\title{
Rapid Prototyping a Semantic Web Application for Cultural Heritage: The Case of MANTIC
}

\author{
Glauco Mantegari, Matteo Palmonari, and Giuseppe Vizzari \\ Department of Computer Science, Systems and Communication (DISCo), \\ University of Milan - Bicocca, \\ via Bicocca degli Arcimboldi 8, 20126 - Milan, Italy \\ \{mantegari, palmonari, vizzari\}@disco.unimib.it
}

\begin{abstract}
MANTIC is a Web application that integrates heterogenous and legacy data about the archeology of Milan (Italy); the application combines semantic Web and mashup technologies. Semantic Web models and technologies supports model-driven and standard-compliant data integration on the Web; the mashup approach supports a spatial and temporal aware form of information presentation. MANTIC shows that model-driven information integration applications for cultural heritage can be fast prototyped with limited deployment effort by combining semantic and mashup technologies. Instead, higher-level modeling aspects need a deep analysis and require domain expertise.
\end{abstract}

\section{Introduction and Motivation}

Semantic Web technologies (SWT) natively provide support for model-based information integration, exchange and processing by offering Web-compliant knowledge representation languages (e.g. RDF, RDFS and OWL), query and reasoning engines. Mashup technologies (MTs) [1] provide support for simple application integration and for aggregating heterogeneous information on the Web. Combining SWTs and MTs it is possible to build data-driven Web applications with limited efforts and costs, by reusing available information sources and models, and information presentation layers. Model-driven information integration, and reuse of application services are particularly attractive in the field of cultural heritage $(\mathrm{CH})$ because of a number of issues that characterize the domain.

First of all, available funds are often limited, in particular when an application is not part of a large national or international project. Second, although a ISO standard conceptual reference model for $\mathrm{CH}$ has been recently developed, i.e. the CIDOC CRM most of data sources are still based on legacy models and non trivial ontology-based mappings are needed in order to support information integration. Furthermore the frequent and heterogeneous spatial and temporal references (e.g. multiple spatial references and different historical classification systems), require reasoning capabilities (e.g. on temporal intervals); such references are crucial to support the access to the information (e.g. by map-based result presentation and timelines rendering), which makes the

\footnotetext{
${ }^{1}$ http://www.lintar.disco.unimib.it:8080/mantic/

${ }^{2}$ http://cidoc.ics.forth.gr/

L. Aroyo et al. (Eds.): ESWC 2010, Part II, LNCS 6089, pp. 406 410, 2010.

(C) Springer-Verlag Berlin Heidelberg 2010
} 


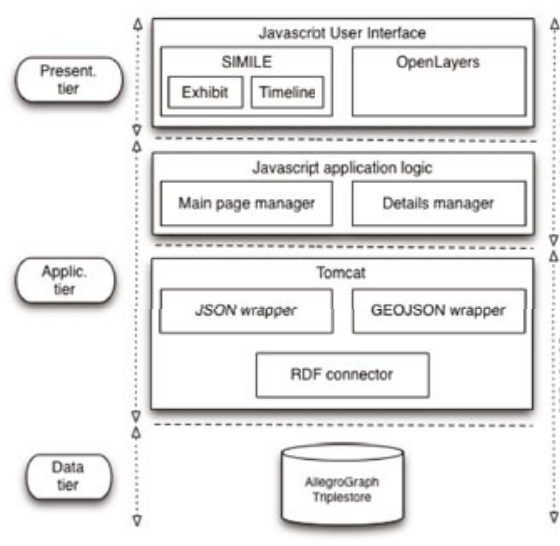

(a)

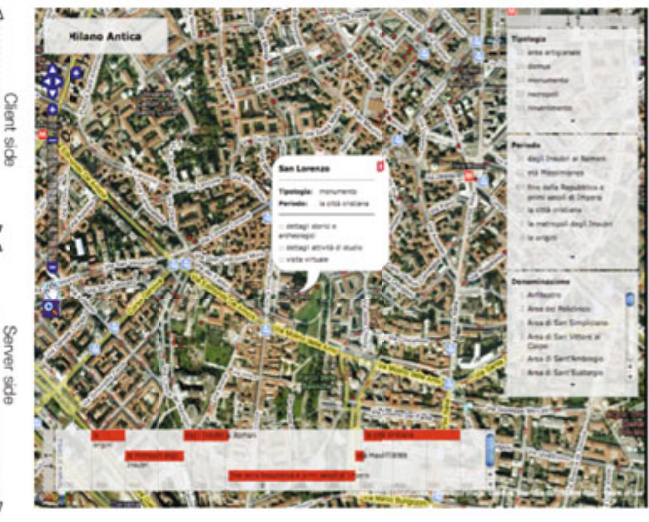

(b)

Fig. 1. Mantic Architecture (a) and a screenshot of the navigation page (b)

reuse external services (e.g. Google Maps) and software components (e.g. the SIMILE 3 timeline representations) particularly useful. Finally, rich and deeply nested descriptions due to the modeling choices of the CIDOC CRM (and the related OWL-DL implementation presented in [2]): the relevant information describing, for instance, a monument is represented as a very nested graph [3] and this requires reasoning engines (e.g. to retrieve data through the subclass chains down to the actual sources) as well as optimization techniques (e.g. because of expensive query answering).

MANTIC is a Web application that, considering the above issues, realizes a portal for archaeological information concerning the city of Milan, which is based on the integration of SWTs and MTs: a Web mashup approach provides browsing and navigation functionalities on a semantic repository integrating legacy information sources (a Web portal and existing relational databases). The mashup approach allowed to reuse available services and software components focusing on a set of core aspects related to the modeling activity. MANTIC shows that by combining SWTs and MTs it is possible to fast prototype model-driven information integration applications with limited deployment effort and budget (the application is based on free software and required 5 person/months of a bachelor-level computer scientist), and focusing on higher-level modeling aspects that require domain expertise (activities of existing data schemas analysis and modeling/mapping definitions required 6 person/months or a domain expert).

The application shows that effective results can be obtained also by exploiting data available from the deep Web and with very limited funds on national or international scale. Actually, most of the relevant works carried out in developing semantic Web applications in the field of $\mathrm{CH}$ comes from european project or equivalent national ones (e.g. eCHASE[4], Culture Sampo[5], STITCH[6], The Vbi Erat Lvpa [7], STAR [8] and Perseus-Arachne [9] projects). Conversely, our application shows the effectiveness of combining MTs and SWTs even in smaller, region-scale contexts.

\footnotetext{
${ }^{3} \mathrm{http}: / /$ simile.mit.edu/
} 
In this paper, we focus on two main classes of outcomes of the project: the integration of legacy and Web sources scraped from the Web, including the information extraction, modeling and mapping processes, discussed in Section 2, the system architecture and functionalities, including the SWTs used, the browsing approach, the timeline navigation, and the optimization techniques needed, discussed in Section 3. Lessons learned are also discussed at the end of the paper (Section 4).

\section{Information Extraction, Modelling and Mapping}

MANTIC integrates the following data sources:

- SIRBeC: the Regional Information System on cultural heritage is the main technological platform created by the Regione Lombardia for cataloging cultural heritage (compliant to and representative of the Italian cataloguing standards); it collects information about the items that are present in the territory or are preserved in museums, collections and other cultural institutions.

- IDRA: the Information Database on Regional Archaeological-Artistic-Architectural heritage which connects several databases related to Lombardia heritage; it collects archaeological data concerning the city of Milan (Italy); these data have been obtained by means of Web scraping techniques.

- MANTIC core: a PostgreSQL database containing synthetic information about the archaeological sites, structures and monuments of the city.

Each of the above data sources comes with a local schema and has been transformed with ETL techniques into a RDFS++ ontology. The global schema is based on the CIDOC CRM standard. In particular the OWL-DL CIDOC CRM representation defined by [2] has been used. The main challenges faced in the process concerned the following aspects:

Event-centric global model vs Object-centric local models. The CIDOC CRM model represented in the global schema substantially differs from the models used in the local sources. In particular, the former adopts an event-centric approach, where object descriptions are represented through descriptions of events related to these objects (e.g. the production, modification, or documentation of a given artifact). On the contrary, local sources adopt an object-centric approach, where descriptions are referred to objects themselves. A significant amount of implicit information had to be identified, analyzed, and represented to leverage this substantial ontological mismatch. The most frequent problems in the design of mappings are related to (i) different subgraphs for equivalent metadata, and (ii) identical subgraphs for different metadata. The graph representing the global schema is thus more composite than the original models; as a consequence complex graph queries are required to retrieve even relatively simple information.

Multiple historical periods reference systems. Different interpretation of the same periods were assumed in different data sources, and multiple classifications of historical periods are present.

Coreference Linking. The integration of complementary sources through a common model is intrinsically characterized by the problem of identifying possible different resource identifiers (e.g. URIs) that refer to the same real world entities. In addition, in 
this context we have a problem of multiple temporal references and different classification systems for historical periods.

\section{System Architecture and Functionalities}

MANTIC architecture is shown in Figure 1. After a comparison of different triple storage we chose to use Allegro Graph 4 because of the good trade-off among the reasoning capabilities offered and efficiency. In particular, Allegro Graph natively support $\mathrm{RDFS}^{++}$, which enrich RDFS reasoning capabilities (necessary to support type inference through subclass relationship) with support for owl:sameas and for inverse and transitive properties (useful respectively to support coreference, and reasoning about temporal intervals). Data obtained by querying the triple store is translated to JSON format (managed by the UI components) by a specific connector.

The main functionalities of MANTIC are:

- Map-Based Browsing: The system presents a view of the Milan area in which the relevant archaeological evidences (e.g. monuments and sites of interest dating between V century B.C. and VI century A.D.) are shown as points in the modern city map. The interactive mapping system is based on the OpenLayers framework 5 ; it currently shows cartographic data fetched from standard Internet mapping services (i.e. Google Maps and Openstreetmap), but the integration and employment of local and more precise data from Regione Lombardia is currently being implemented.

- Faceted Browsing and Timeline based filtering: The system presents two types of additional UI elements that can be employed to filter the shown monuments and sites: a timeline supporting the selection of the elements according to the different phases in which the relevant time period is subdivided, and facets highlighting typological characterizations (e.g. domus, monument, necropolis) and possibly other relevant dimensions of cultural heritage description (e.g. materials, building techniques, etc.).

- Query Optimization and Caching: The first approaches to the definition of SPARQL queries to retrieve information from the Allegro Graph triple store were unsatisfactory: given the peculiarities of the CIDOC CRM even conceptually simple queries (e.g. retrieving basic information about monuments), required to retrieve and filter information related to several CIDOC classes, leading to moderately complex queries whose execution would not be compatible with the expected time delay for a dynamic web page generation. To tackle this issue we decided to (i) adopt caching techniques to avoid unnecessary query processing, and (ii) translated complex queries into equivalent portions of Java code employing simpler queries.

\section{Lessons Learned and Conclusions}

The combination of SWTs and MTs supports the rapid prototyping of CH Web applications for the integration and presentation of heterogeneous information from legacy

\footnotetext{
${ }^{4}$ http://www.franz.com/agraph/

${ }^{5} \mathrm{http}$ ://openlayers.org
} 
sources with limited development efforts. In model-driven contexts such as $\mathrm{CH}$, particular attention must be instead paid to modeling issues due to sensible ontological mismatches among heterogenous models. In particular, the role of domain experts with respect to the definition of mappings from heterogeneous documentation systems to the CRM becomes crucial. In the specific project, this modeling effort will simplify the integration of further information sources complying to Italian cataloguing standards. In developing such an application we experienced a trade-off between model-level qualities like richness and generality of a standard ontology (i.e. the CIDOC CRM in our case), and application-level qualities such as efficiency and simplicity. Finally, we also noticed that RDFS++ presents a good balance between reasonable scalability and useful reasoning task support for information integration and navigation.

\section{References}

1. Yu, J., Benatallah, B., Casati, F., Daniel, F.: Understanding Mashup Development. IEEE Internet Computing 12(5), 44-52 (2008)

2. Goerz, G., Schiemann, B., Oischinger, M.: An Implementation of the CIDOC Conceptual Reference Model (4.2.4) in OWL-DL. In: CIDOC Annual Conference, September 1518 (2008), http://www8.informatik.uni-erlangen.de/IMMD8/Services / cidoc-crm/docu/crm_owl_cidoc2008.pdf

3. Nussbaumer, P., Haslhofer, B.: CIDOC CRM in Action - Experiences and Challenges. In: Kovács, L., Fuhr, N., Meghini, C. (eds.) ECDL 2007. LNCS, vol. 4675, pp. 532-533. Springer, Heidelberg (2007)

4. Addis, M.J., Hafeez, S., Prideaux, D., Lowe, R., Lewis, P.H., Martinez, K., Sinclair, P.A.S.: The echase system for cross-border use of european multimedia cultural heritage content in education and publishing. In: AXMEDIS 2006: 2nd International Conference on Automated Production of Cross Media Content for Multi-channel Distribution (2006)

5. Hyvönen, E., Mäkelä, E., Kauppinen, T., Alm, O., Kurki, J., Ruotsalo, T., Seppälä, K., Takala, J., Puputti, K., Kuittinen, H., Viljanen, K., Tuominen, J., Palonen, T., Frosterus, M., Sinkkilä, R., Paakkarinen, P., Laitio, J., Nyberg, K.: CultureSampo: A National Publication System of Cultural Heritage on the Semantic Web 2.0. In: Aroyo, L., et al. (eds.) ESWC 2009. LNCS, vol. 5554, pp. 851-856. Springer, Heidelberg (2009)

6. van Gendt, M., Isaac, A., van der Meij, L., Schlobach, S.: Semantic web techniques for multiple views on heterogeneous collections: A case study. In: Gonzalo, J., Thanos, C., Verdejo, M.F., Carrasco, R.C. (eds.) ECDL 2006. LNCS, vol. 4172, pp. 426-437. Springer, Heidelberg (2006)

7. Doerr, M., Schaller, K., Theodoridou, M.: Integration of complementary archaeological sources. In: Proceedings of the 32nd Computer Applications and Quantitative Methods in Archaeology Conference (2004)

8. Binding, C., May, K., Tudhope, D.: Semantic Interoperability in Archaeological Datasets: Data Mapping and Extraction Via the CIDOC-CRM. In: Christensen-Dalsgaard, B., Castelli, D., Ammitzbøll Jurik, B., Lippincott, J. (eds.) ECDL 2008. LNCS, vol. 5173, pp. 280-290. Springer, Heidelberg (2008)

9. Babeu, A., Bamman, D., Crane, G., Kummer, R., Weaver, G.: Named Entity Identification and Cyberinfrastructure. In: Kovács, L., Fuhr, N., Meghini, C. (eds.) ECDL 2007. LNCS, vol. 4675, pp. 259-270. Springer, Heidelberg (2007) 